\title{
Análise da produção de conhecimento da Educação Física brasileira sobre o cotidiano escolar
}

\author{
Analysis of the Brazilian production of knowledge \\ of Physical Education on the daily school
}

\begin{abstract}
Análisis de la producción de conocimiento de la Educación Física brasileña sobre el cotidiano escolar.
\end{abstract}

Renato Xavier Coutinho, doutorando e mestre em Educação em Ciências pela Universidade Federal de Santa Maria. Endereço: Avenida Roraima, $n^{\circ}$ 1000, prédio 13B, Campus Universitário Camobi. CEP: 97105-900 - Santa Maria, RS. Telefone: (55) 81115670. E-mail: renatocoutinho@msn.com.

Max Castelhano Soares, doutorando e mestre em Educação em Ciências pela Universidade Federal de Santa Maria. Endereço: Avenida Roraima, $n^{\circ}$ 1000, prédio 13B, Campus Universitário Camobi. CEP: 97105-900 - Santa Maria, RS. Telefone: (55) 34134321. E-mail:maxcastelhano@yahoo.com.br.

Vanderlei Folmer, professor adjunto doutor na Universidade Federal do Pampa, Campus Uruguaiana. Endereço: BR 472, Km 7. CEP: 97500-970 - Uruguaiana, RS. Telefone: (55) 3413-4321. E-mail: vanderleifolmer@unipampa.edu.br.

Robson Luiz Puntel, professor adjunto doutor na Universidade Federal do Pampa, Campus Uruguaiana. Endereço: BR 472, Km 7. CEP: 97500-970 - Uruguaiana, RS. Telefone:(55) 3413-4321. Email: robson_puntel@yahoo.com.br. 


\section{Resumo}

O objetivo deste estudo foi analisar a produção de conhecimento da Educação Física brasileira relacionada ao contexto escolar. Para tanto, foram analisados grupos de pesquisa registrados no CNPq e programas de pós-graduação, teses, dissertações e artigos científicos em periódicos com classificação superior a B2 na área. A análise revelou uma grande expansão em todos os parâmetros analisados, principalmente a partir do ano 2000, sendo que foram identificados 211 grupos de pesquisa, 22 programas de pós-graduação, dois periódicos B1 e seis com Qualis B2, totalizando 242 artigos. Contudo, apesar do evidente aumento da produção científica, são necessárias medidas para disseminar o conhecimento gerado. Assim, espera-se que este estudo possa, ao demonstrar o que está sendo feito pela Educação Física voltado ao contexto escolar, apontar caminhos para a melhoria dessa produção acadêmica.

Palavras-chave: Educação Física. Escola. Produção Científica.

\section{Abstract}

The aim of this study was to analyze the production of knowledge of the Brazilian Physical Education related to the school context. It was analyzed the research groups registered in the CNPq, post graduate Stricto Sensu programs, theses, dissertations and papers in journals that are ranked higher $\mathrm{B} 2$ in the area. The analysis revealed a great expansion, in all the studied parameters, mainly from 2000, of which were identified 211 research groups, 22 graduate programs, 2 journals B1 and 6 with Qualis B2, totalizing 242 papers. However, despite the evident increase of the scientific production, it is necessary to disseminate the knowledge generated. Thus is expected that this study may, by demonstration of what is being done by the Physical Education focused on school context, point the way to the improvement of their academic output.

Keywords: Physical Education. School. Scientific Production. 


\section{Resumen}

El objetivo de este estudio fue analizar la producción de conocimiento de la Educación Física brasileña relacionada con el contexto escolar. Para ello, fueron analizados grupos de investigación registrados en el CNPq (Consejo Nacional de Desarrollo Científico y Tecnológico) y programas de posgrado, tesis de Doctorado, trabajos finales de Maestría y artículos científicos en periódicos con clasificación superior a B2 en el área. El análisis reveló una gran expansión en todos los parámetros analizados, principalmente a partir del año 2000, siendo que fueron identificados 211 grupos de investigación, 22 programas de posgrado, dos periódicos B1 y seis con Qualis B2, totalizando 242 artículos. Sin embargo, a pesar del evidente aumento de la producción científica, son necesarias medidas para diseminar el conocimiento generado. De esta manera, se espera que este estudio pueda, al demostrar lo que está siendo realizado por la Educación Física volcado al contexto escolar, apuntar caminos para la mejoría de esta producción académica.

Palabras clave: Educación Física. Escuela. Producción Científica.

\section{Introdução}

A presença da Educação Física nas práticas escolares brasileiras remonta ao século XIX e, desde então, experimenta um processo permanente de enraizamento (VAGO, 1999). No entanto, apenas em 1929 ocorre a formação da primeira turma do primeiro curso de Educação Física no Centro Militar de Educação Física no Brasil, com 20 professores civis (BAPTISTA et al., 2002). Atualmente, segundo o Ministério da Educação (MEC), existem aproximadamente 800 cursos de Educação Física no País. De particular importância, Melo (1996) constata que a esse aumento associam-se preocupações relativas à história da Educação Física, refletindo diretamente sobre o aumento da sua produção científica. No entanto, percebem-se poucas discussões e reflexões relativas à qualidade, à natureza e às especificidades dessa produção.

Paralelamente a essa expansão dos cursos de graduação, ocorreu um incremento no número de grupos de pesquisa na área da Educação Física registrados no Conselho Nacional de Desenvolvimento Científico e Tecnológico (CNPq). Da mesma forma, há um grande número 
de teses, dissertações e artigos sendo produzidos continuamente nos programas de pós-graduação. Daolio (2007) afirma que, com isso, a área de Educação Física deixa de ser apenas uma área de prática pedagógica ou de aplicação de conceitos provenientes de outras áreas acadêmicas para tornar-se área de produção de conhecimento científico. Percebe-se também, nessa área do conhecimento, a existência de duas vertentes: 1) as pesquisas amparadas nas Ciências Naturais e Exatas, que buscam efeitos e resultados do treinamento e da atividade física sobre os perfis antropométricos, capacidade cardiorrespiratória e capacidade funcional, além do rendimento esportivo; e 2) as pesquisas que têm relação com as Ciências Sociais e Humanas relativas ao contexto escolar e ao movimento humano, que trabalham os conteúdos didáticos e pedagógicos que permeiam a Educação Física no ambiente escolar.

Nesse contexto, o conhecimento dos rumos da produção científica de uma área é fundamental para a melhoria da qualidade da pesquisa, para os avanços da ciência e, principalmente, para o diagnóstico do impacto dessa produção no âmbito social de sua criação. A busca de explicações sobre os caminhos adotados pela pesquisa permite evidenciar suas conquistas, indicar suas lacunas e, ao mesmo tempo, chamar atenção para novas alternativas de investigações (MARTINS; SILVA, 2009).

Desse modo, estudos e pesquisas sobre os indicadores científicos são fundamentais para o crescimento e a reflexão em torno das práticas dessa área, pois, desde que a Educação Física foi pensada enquanto disciplina nas escolas brasileiras, teve propósitos profiláticos morais e culturais. Embora Educação Física e Educação Física escolar pareçam ser a mesma coisa, não são sinônimos, já que a escola é um dos ramos profissionais do educador físico (BELTRAMI, 2001). Ademais, os conteúdos relacionados ao cotidiano escolar compreendem uma das possibilidades de atuação dos educadores físicos (TANI, 2000).

Como em qualquer outra área, o conhecimento produzido pela Educação Física deveria repercutir na melhoria da prática dos seus profissionais em todas as possibilidades de atuação, ou seja, nas academias, nos clubes, nos grupos de atividade física e, principalmente, nas escolas. Sobral (2009) descreve que as universidades brasileiras 
vivenciam na atualidade um grande desafio: o de atender aos requisitos de qualidade como mérito acadêmico da sua produção científica e tecnológica e de relevância social e econômica. Entretanto, como fazer para que o conhecimento produzido nas instituições de ensino superior chegue até os professores nas escolas e atenda às demandas da sociedade, para que não seja estéril, fique ao alcance de poucos ou, ainda, restrito aos que o produzem? Carvalho (2002) entende que existe uma distância que separa o discurso acadêmico-científico das universidades do cotidiano das escolas públicas e que isso enseja vários questionamentos.

Assim, refletir sobre o quanto e como discutimos a Educação Física escolar se faz necessário. De fato, conforme Falcão (2007), a produção acadêmica da Educação Física, embora expressiva, continua pautada em sua maioria em modelos distantes da cultura popular. Da mesma forma, Caparroz e Bracht (2007) compreendem que é importante o aprofundamento das reflexões em torno dos processos de ensino-aprendizagem e do debate sobre os fundamentos didáticos e pedagógicos da Educação Física, que têm sido negligenciados nos últimos anos.

Portanto, a partir do problema exposto, este estudo teve o objetivo principal de analisar a produção científica da Educação Física voltada ao contexto escolar, efetuando um diagnóstico acerca das áreas de concentração dos programas de pós-graduação stricto sensu, das teses e dissertações, dos grupos registrados no CNPq e dos artigos publicados em periódicos de divulgação científica. Além disso, foram identificados aspectos tais como: distribuição geográfica dos grupos, pesquisadores e estudantes envolvidos, linhas de pesquisa e assuntos abordados.

\section{Métodos}

A busca pelos dados da produção de conhecimento na área de Educação Física estruturou-se em quatro aspectos: grupos e linhas de pesquisa registrados no CNPq; programas de pós-graduação stricto sensu da área da Educação Física recomendados pela Coordenação 
de Aperfeiçoamento de Pessoal de Nivel Superior (Capes); teses e dissertações disponíveis no Banco de Teses e Dissertações da Capes; e artigos em periódicos de divulgação científica.

Para obter informações sobre grupos e linhas de pesquisa, foi utilizado o diretório de grupos do CNPq, que possui informações sobre os grupos de pesquisa em atividade no País. As informações contidas nessa base dizem respeito aos recursos humanos constituintes dos grupos (pesquisadores, estudantes e técnicos), às especialidades do conhecimento, às linhas de pesquisa, à região, à instituição e ao tempo de formação.

Quanto aos dados relativos aos programas de pós-graduação stricto sensu da Educação Física recomendados pela Capes, foram realizadas visitas aos sítios eletrônicos desses programas e foi utilizado - Banco de Teses da Capes para a coleta de informações sobre teses e dissertações.

Em relação aos periódicos da Educação Física, foram utilizados dois critérios para a escolha: estar vinculado a programas de pósgraduação stricto sensu da Educação Física e apresentar classificação B2 ou superior no sistema Qualis/Capes. Assim, após a visita ao site do referido sistema, foram selecionados os seguintes periódicos: com Qualis B1, Motriz: Revista de Educação Física (on-line) e revista Movimento; com avaliação B2, Revista Brasileira de Atividade Física e Saúde; Revista Brasileira de Ciência e Movimento; Revista Brasileira de Ciências do Esporte; Revista Brasileira de Cineantropometria \& Desempenho Humano; Revista Brasileira de Educação Física e Esporte; e Revista da Educação Física/UEM). Ademais, a preferência por periódicos nacionais se deu após a análise dos currículos dos pesquisadores bolsistas de produtividade do CNPq, quando ficou constatado que a maioria dos pesquisadores da área da Educação Física publica seus trabalhos em periódicos nacionais.

Além disso, foi analisado o número de artigos publicados em bases de dados internacionais relativos à temática Educação Física escolar. Para tanto, foi acrescentado às palavras-chaves "Brazil”, com o intuito de delimitar a busca. As bases internacionais visitadas foram: PubMed, ScienceDirect, Scirus, ISI Web of Knowledge e Scopus. 
A pesquisa dos dados dos grupos de pesquisa, dos programas de pós-graduação, das teses, das dissertações e dos artigos ocorreu utilizando como palavras-chave "Educação Física” e "escola”, escolhidas após testes com diversas palavras referentes ao contexto escolar, pois essas foram as que apresentaram um maior número de ocorrências de resultados com menores índices de falso-positivos. Foram coletados todos os dados disponíveis entre 1987 e 2010.

A análise dos dados foi realizada por meio da leitura dos resumos das teses, das dissertações e dos artigos; após essa etapa, ocorreu a classificação, utilizando uma matriz analítica baseada em Marzari e Acosta (2007), por meio da qual foi possivel categorizar a produção de conhecimento em diversos aspectos, tais como: método (quantitativo ou qualitativo), em relação às ciências naturais exatas e sociais humanas e aos paradigmas de pesquisa (empírico-analítico, fenomenológico-hermenêutico e crítico-dialético, segundo Souza e Silva, 1990); distribuição geográfica dos grupos; pesquisadores e estudantes envolvidos; linhas de pesquisa; e assuntos abordados.

Em relação à classificação por paradigmas de pesquisa, optou-se pelo ordenamento elaborado por Souza e Silva (1990); apesar de não ser uma classificação nova, ainda é bastante utilizada quando se trata da produção de conhecimento da Educação Física. No entanto, conforme Quintão e Vaz (2010) ressaltam, esses paradigmas de pesquisa não são os únicos da epistemologia da Educação Física. Essa categorização descreve os paradigmas da seguinte maneira: a abordagem empírico-analítica pressupõe a "objetividade" pela centralização do processo cognitivo no objeto, ressaltando a imparcialidade do pesquisador. Enfatiza a relação causal que se explicita no experimento, na sistematização, no controle dos dados empíricos e por meio de análises estatísticas. De certa forma, segue os princípios válidos para as ciências físicas e naturais. Fundamenta a validação científica no teste dos instrumentos de coleta e tratamento dos dados e no grau de significância estatística. Privilegia a concepção funcionalista de homem. Já a perspectiva fenomenológicohermenêutica defende a "subjetividade" decorrente da centralização do processo cognitivo no sujeito. Expressa interesse específico na denúncia e no desvelamento das ideologias subjacentes; analisa obras, palavras, gestos, ações, textos, símbolos e discursos que precisam 
ser desvendados em seu sentido, enfatizando que a compreensão pressupõe uma interpretação, o conhecimento do significado que não se dá imediatamente. O critério de cientificidade baseia-se no processo lógico de interpretação e na capacidade de reflexão do pesquisador sobre o fenômeno estudado. Predomina a visão existencial do homem. Por fim, os estudos crítico-dialéticos analisam os problemas, educacionais ou outros, de forma rigorosa, a partir da sua realidade no contexto das relações sociais próprias do modo de produção capitalista. Reconhece a incidência do aspecto ideológico e filosófico em todo o processo investigativo, em seu conjunto, e, portanto, nas etapas empíricas de coleta, processo e análise. Considera o homem como ser social e histórico.

O exame ocorreu exclusivamente por meio eletrônico na World Wide Web, pois, segundo Smith (apud VANTI, 2002), as pesquisas on-line apresentam uma série de vantagens quando comparadas com outros "caminhos de busca", pois permitem trabalhar com grandes volumes de informações que podem ser estáveis, diminuírem, aumentarem ou até mesmo desaparecerem, facilitando as tarefas de quantificação e avaliação dos fluxos de intercâmbio de dados, apresentando vantagens em comparação com as citações científicas impressas de revistas, de instituições ou de indivíduos.

Além disso, conforme Prado, Amorim e Abreu (2003), a organização, a sistematização e a disseminação de informações por meio da implementação de bases de dados eletrônicas que contemplem as mais diversas áreas temáticas de conteúdo permitem o estabelecimento de vínculos com instituições afins, buscando integrar redes de informação e conhecimento. Moutinho e Cunha Filho (2002) descrevem que o suporte digital é exatamente aquele que proporciona o tipo de publicação eletrônica e que tem aparecido em número crescente e sobre os mais diferentes tipos de informação, como textos acadêmicos e literários. 


\section{Resultados e discussão}

\section{Grupos de pesquisa}

O período compreendido no estudo, em relação aos grupos de pesquisa, foi das séries históricas, que consistem em relatórios e pesquisas realizadas pelo CNPq dentro do Diretório dos Grupos de Pesquisa, que analisam sua evolução e seu desenvolvimento até a base de 2010. Todo o levantamento de dados foi realizado no período entre março e maio de 2010. Foi encontrada uma grande expansão no número de grupos de pesquisa que investigam sobre educação na área da Educação Física, passando de 33 no ano 2000 para 211 grupos em 2010 (Tabela 1). Desses, 44 grupos (20,85\%) estão vinculados a programas de pós-graduação stricto sensu. Entretanto, alguns grupos de pesquisa apresentaram apenas pesquisadores cadastrados, sem a participação de estudantes.

Tabela 1. Expansão dos grupos de pesquisa distribuídos por região (contribuição de cada região no período considerado em \%)

\begin{tabular}{l|r|r|r|r|r}
\hline Região & \multicolumn{1}{c|}{$\mathbf{2 0 0 0}$} & \multicolumn{1}{c|}{$\mathbf{2 0 0 2}$} & \multicolumn{1}{c|}{$\mathbf{2 0 0 4}$} & \multicolumn{1}{c|}{$\mathbf{2 0 0 6}$} & \multicolumn{1}{c}{$\mathbf{2 0 1 0}$} \\
\hline Centro-Oeste & $2(6 \%)$ & $3(4 \%)$ & $8(9 \%)$ & $11(09 \%)$ & $22(10 \%)$ \\
\hline Nordeste & $2(6 \%)$ & $6(0 \%)$ & $14(15 \%)$ & $19(16 \%)$ & $34(16 \%)$ \\
\hline Norte & - & - & $1(1 \%)$ & $2(2 \%)$ & $8(4 \%)$ \\
\hline Sudeste & $17(51 \%)$ & $28(40 \%)$ & $36(37 \%)$ & $44(38 \%)$ & $76(36 \%)$ \\
\hline Sul & $12(37 \%)$ & $34(48 \%)$ & $37(38 \%)$ & $41(35 \%)$ & $71(34 \%)$ \\
\hline TOTAL & $\mathbf{3 3}$ & $\mathbf{7 1}$ & $\mathbf{9 6}$ & $\mathbf{1 1 7}$ & $\mathbf{2 1 1}$ \\
\hline
\end{tabular}

O estudo demonstrou uma grande concentração de grupos registrados no CNPq nos estados das regiões Sul e Sudeste. O estado de São Paulo, conforme Baptista et al. (2002), possuía na época 31,5\% dos cursos de Educação Física do País, o que explica em parte a hegemonia da região Sudeste no que se refere ao maior número de grupos de pesquisa. Acompanhando o mesmo raciocínio, Lüdorf (2002), ao estudar os resumos de teses e dissertações dos cursos de pós-graduação em Educação Física na década de 1990, encontrou uma concentração de $76 \%$ na região Sudeste e $24 \%$ na região Sul, enquanto as outras regiões 
não apresentaram trabalhos. Em uma pesquisa semelhante envolvendo grupos de pesquisa da Educação Física que investigaram a prática do lazer, Souza e Isayama (2006) também encontraram maior presença de grupos na região Sudeste (66\%), seguida pelas regiões Sul $(25 \%)$ e Nordeste $(11 \%)$.

Dos 211 grupos de pesquisa investigados, 121 apresentaram linhas de pesquisa específicas sobre temas relativos ao contexto escolar. Nesses 121 grupos, foram encontradas 404 linhas de pesquisa, sendo que 202 (50\%) eram referentes à escola. Ao analisar as linhas de pesquisa, observou-se um predomínio daquelas referentes a aspectos pedagógicos e de formação de professores. Foram identificadas ainda aquelas relativas a: esporte, saúde e qualidade de vida, lazer, corporeidade e cultura corporal, memória e história da Educação Física, Educação Física para pessoas com necessidades especiais, desenvolvimento motor e aprendizagem motora, cineantropometria, medidas e avaliação, treinamento desportivo, envelhecimento humano, produção de conhecimento e epistemologia, gênero e biomecânica.

Considerando apenas os grupos com linhas de pesquisa específicas, encontramos 370 (46\%) homens e 431 (54\%) mulheres; em relação aos estudantes, também observamos um predomínio de mulheres (537 ou 61\%) sobre os homens (348 ou 39\%). Em nenhuma região houve predomínio de homens, o que reforça a ideia do aumento no número de mulheres nas diferentes áreas do conhecimento (LETA, 2003).

Entre as instituições, há uma prevalência de universidades públicas pesquisando sobre o tema, sendo que $74 \%$ dos grupos de estudo com linhas específicas registradas são de instituições públicas, enquanto $26 \%$ são de instituições privadas. Esse dado acompanha o panorama atual dos cursos de pós-graduação em nível de mestrado e doutorado, dado que há uma prevalência maior de instituições públicas sobre as privadas com relação à pós-graduação no Brasil em todas as áreas de conhecimento (DE MEIS; ARRUDA; GUIMARÃES, 2007). De acordo com Sousa (2009), as instituições de ensino privadas preocupam-se mais com a lógica da concorrência de mercado, oferecendo cursos no nível de graduação em que a demanda é maior, enquanto que as públicas 
investem mais em pós-graduação e pesquisa e, consequentemente, na produção de conhecimento.

O grupo de pesquisa mais antigo registrado no diretório de grupos do CNPq é o Grupo de Pesquisa de Educação Física e Motricidade Humana, da Pontifícia Universidade Católica de São Paulo, criado em 1989. As instituições com maior número de grupos, com linhas de estudo específicas, foram a Universidade Estadual de Londrina (UEL), Universidade Estadual de Campinas (Unicamp) e a Universidade Federal de Santa Maria (UFSM), com sete grupos cada. O estado com o maior número de grupos foi o de São Paulo, com 27. Esses dados estão de acordo com Amadio (2003), que descreveu em seu trabalho que a grande maioria dos programas de mestrado $(62 \%)$ e de doutorado $(79 \%)$ e quase $80 \%$ dos alunos matriculados na pós-graduação do Brasil concentram-se nos estados da região Sudeste, principalmente em São Paulo.

O grande número de assuntos encontrados nas linhas de pesquisa demonstra, conforme Tani (2000), que as bases epistemológicas da Educação Física são muito heterogêneas, o que não causa estranheza devido ao variado campo de atuação dos professores de Educação Física. Gamboa, Chaves e Taffarel (2007) citam, quanto às abordagens utilizadas nas investigações da área, uma tendência de diminuição das pesquisas analíticas e positivistas e um aumento das pesquisas crítico-dialéticas e fenomenológicas.

\section{Programas de pós-graduação na área de Educação Física}

Martins e Silva (2009) apontam que até 2003 a Capes recomendava apenas 13 programas de pós-graduação em Educação Física, sendo que em nossa busca encontramos 22 programas (Tabela 2). O presente estudo revelou ainda que os focos dos programas de pós-graduação stricto sensu na área de Educação Física concentramse, em sua maioria, nos aspectos biodinâmicos do movimento humano relacionados à atividade física e à saúde (78\%) e nos aspectos pedagógicos do movimento humano em menor grau $(22 \%)$.

Quanto à distribuição geográfica dos programas (Tabela 2), notouse que a região que possui maior número de programas é a Sudeste, 
seguida pela Sul, Centro-Oeste e Nordeste - não foram encontrados programas na região Norte. 0 estado de São Paulo foi o que apresentou o maior número de programas (sete de mestrado e três de doutorado). Da mesma forma, o programa de pós-graduação há mais tempo em atividade está na Universidade de São Paulo (USP), cujo mestrado foi criado em 1977 e o doutorado em 1989.

Tabela 2. Distribuição geográfica dos programas de pós-graduação

\begin{tabular}{l|c|c}
\hline Estado & Mestrado & Doutorado \\
\hline São Paulo & 7 & 3 \\
\hline Rio de Janeiro & 3 & 1 \\
\hline Santa Catarina & 2 & 1 \\
\hline Paraná & 2 & 1 \\
\hline Rio Grande do Sul & 2 & 1 \\
\hline Minas Gerais & 2 & 1 \\
\hline Distrito Federal & 2 & - \\
\hline Pernambuco & 1 & - \\
\hline Espírito Santo & 1 & \\
\hline
\end{tabular}

O programa da Universidade de São Paulo é também o que atualmente apresenta os melhores conceitos (6 para mestrado e doutorado, notas máximas concedidas pela Capes para Educação Física). Analisando todos os programas, encontramos uma concentração de $45 \%$ deles com conceito $3,23 \%$ conceito 4 e $23 \%$ conceito 5 . Provavelmente, isso pode estar relacionado ao fato de que a maioria dos programas foi criada há menos de 10 anos e ainda não está suficientemente estabelecida. Além disso, encontramos, ao analisar os relatórios de avaliação da pós-graduação da Capes (2010) que, quanto mais antigo o programa, melhor tem sido o seu conceito.

Quanto às instituições, as universidades públicas são os grandes centros de produção do conhecimento no que diz respeito à Educação Física, respondendo por $74 \%$ dos programas, enquanto as universidades privadas possuem apenas $26 \%$. De fato, Gomes (2007) assinala que os principais centros de excelência em pesquisa no Brasil estão localizados nas universidades públicas. Em um estudo sobre a produção científica brasileira na área da educação, Carvalho, Tomazello e Oliveira (2009) 
corroboram essa ideia, apontando que o sistema universitário público e, principalmente, a pós-graduação constitui-se em um espaço privilegiado dessa produção.

Das 81 linhas de pesquisa dos programas de pós-graduação, $65 \%$ estão voltadas a estudos referentes ao tema atividade física e saúde, enquanto $24 \%$ estão ligadas a aspectos pedagógicos e didáticos do movimento humano - apenas $11 \%$ estão ligadas a estudos sobre aspectos sociológicos e de memória do esporte. A respectiva sequência de objetos de estudo também foi identificada nos grupos de pesquisa registrados no CNPq, o que vem a confirmar as tendências de pesquisa na Educação Física, que, historicamente, estão mais próximas das tendências positivistas de estudo. Feron e Moraes e Silva (2007) atribuíram essa identificação da Educação Física, com estudos amparados nas Ciências Naturais e Exatas, ao fato de que muitos pesquisadores responsáveis pela produção de conhecimento da área têm sua formação ligada à fisiologia, aprendizagem motora ou biomecânica.

\section{Teses e dissertações}

O Banco de Teses da Capes foi escolhido para a análise das teses e dissertações, pois muitos dos programas de pós-graduação não disponibilizam nos seus respectivos endereços eletrônicos a produção acadêmica de seus cursos. Além disso, pode se inferir que essa seja a base de dados mais completa, pois concentra trabalhos de todas as instituições no período de 1987 até 2008, inclusive daquelas que já tiveram programas de mestrado e doutorado, mas que hoje não os têm mais.

Foi encontrado um total de 430 dissertações e 43 teses sobre Educação Física escolar, porém, desse total, apenas 52,3\% das dissertações e 44,2\% das teses corresponderam ao critério de terem sido elaboradas em programas stricto sensu da Educação Física (Tabela 3). 
Tabela 3. Banco de Teses Capes (1987-2008)

\begin{tabular}{l|r|r|r}
\hline Assunto & Dissertações & \multicolumn{1}{|c|}{ Teses } & \multicolumn{1}{c}{ Total } \\
\hline Educação & 29.407 & 6.293 & 35.700 \\
\hline Educação Física* & 1.838 & 298 & 2.136 \\
\hline Educação Física Escolar & 430 & 43 & 473 \\
\hline Educação Física Escolar** & 225 & 19 & 244 \\
\hline $\begin{array}{l}\text { *Educação Física em sentindo amplo, todas as áreas de pesquisa relacionadas à área. } \\
\text { **Programas de Educação Física. }\end{array}$
\end{tabular}

Sobre os índices de produção científica, encontramos uma grande expansão da produção de teses e dissertações a partir do ano 2000 (Figura 1), o que pode estar vinculado ao aumento no número de grupos de pesquisa registrados no CNPq (Tabela 1) e também no número de programas de mestrado e doutorado em Educação Física recomendados pela Capes. Gomes (2007) aponta ainda a firmação de convênios entre universidades, seja do próprio País ou do exterior, como fator preponderante para o aumento dos índices de produção científica de uma determinada área.

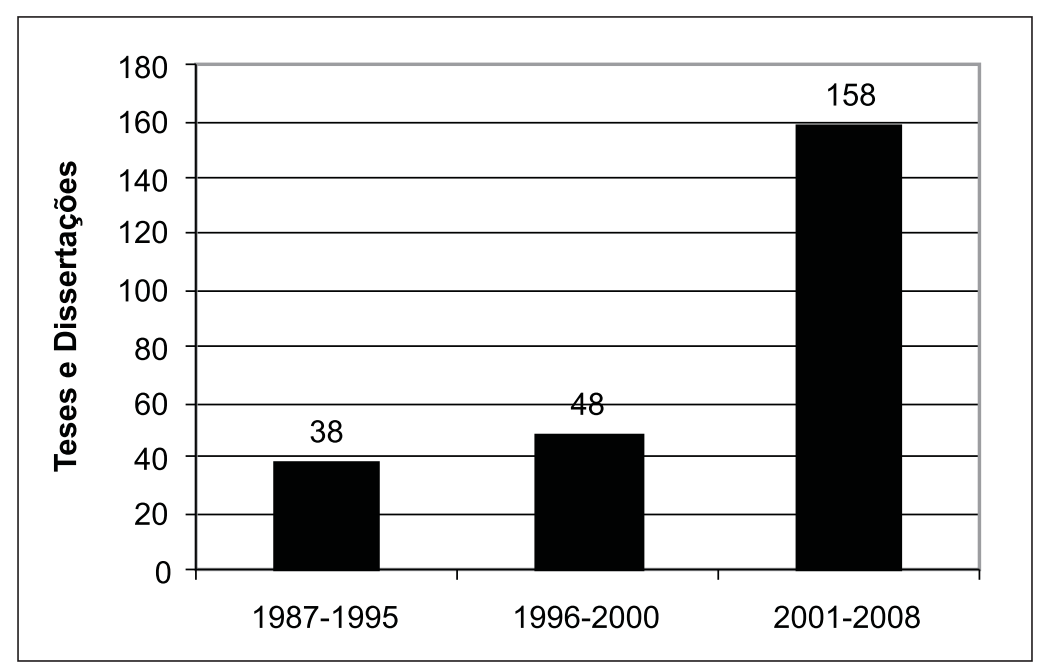

Figura 1. Produção de teses e dissertações

A região Sudeste apresentou a maior concentração de produção acadêmica, seguida pela região Sul e pela região Centro-Oeste, enquanto as regiões Nordeste e Norte não apresentaram trabalhos que preenchiam os critérios de pesquisa (Tabela 4). 
Tabela 4. Dissertações e teses por IES - 1987/2008

\begin{tabular}{l|r|r|r}
\hline \multicolumn{1}{c|}{ INSTITUIÇÕES DE ENSINO SUPERIOR } & Dissertações & \multicolumn{1}{|c|}{ Teses } & \multicolumn{1}{c}{ Total } \\
\hline Universidade Estadual de Campinas & 42 & 10 & 52 \\
\hline Universidade Gama Filho & 40 & 5 & 45 \\
\hline Universidade Federal do Rio Grande do Sul & 13 & 3 & 16 \\
\hline Universidade de São Paulo & 19 & 1 & 20 \\
\hline Universidade Estadual de São Paulo Campus & 32 & - & 32 \\
Rio Claro & 26 & - & 26 \\
\hline Universidade Federal de Santa Catarina & 18 & - & 18 \\
\hline Universidade Metodista de Piracicaba & 13 & - & 13 \\
\hline Universidade Federal de Santa Maria & 5 & - & 5 \\
\hline Universidade do Estado de Santa Catarina & 5 & - & 5 \\
\hline Universidade Católica de Brasília & 5 & - & 5 \\
\hline Universidade São Judas Tadeu & 3 & - & 3 \\
\hline Universidade Castelo Branco & 2 & - & 2 \\
\hline Universidade Federal de Minas Gerais & 1 & - & 1 \\
\hline Universidade de Brasília & 1 & - & 1 \\
\hline Universidade Salgado Filho & $\mathbf{2 2 5}$ & $\mathbf{1 9}$ & $\mathbf{2 4 4}$ \\
\hline TotaL & &
\end{tabular}

Lüdorf (2002), analisando 524 resumos de teses e dissertações defendidas na década de 1990 nos cursos de mestrado e doutorado em Educação Física quanto à abordagem metodológica, encontrou: paradigma empírico-analítico - 296 (56,5\%); paradigma fenomenológicohermenêutico - 220 (42\%); e paradigma crítico-dialético - 8 (1,5\%), demonstrando que as pesquisas em Educação Física de modo geral têm demonstrado uma propensão ao paradigma empírico-analítico.

Recentemente, Martins e Silva (2009), em um estudo sobre teses e dissertações da Educação Física, encontraram como principais resultados: crescimento do número de programas, diversificação das áreas de concentração e linhas de pesquisa e reorientação metodológicoepistemológica da produção científica da área. Além disso, as pesquisas realizadas já não se restringem aos estudos experimentais ou a outros modelos alicerçados na concepção positivista de ciência (MARTINS; SILVA, 2009). Contudo, alguns antigos problemas ainda não foram resolvidos, como o caráter meramente descritivo limitado à constatação dos fatos, o qual ainda é predominante nas pesquisas, mesmo que fundamentadas em referenciais teóricos diferentes de acordo com as autoras. 
A partir da análise das teses e dissertações, pode-se constatar um movimento diferenciado da produção de conhecimento em Educação Física voltada para a escola, pois se em estudos anteriores (LÜDORF, 2002; MARTINS; SILVA, 2009), que abordavam a pesquisa da área da Educação Física como um todo (partindo de teses e dissertações), eram encontrados trabalhos predominantemente fundamentados no paradigma empírico-analítico de cunho quantitativo; já neste estudo encontramos diferenças no que se refere aos paradigmas de pesquisa, pois houve uma supremacia dos estudos de caráter fenomenológico e crítico, correspondendo a $84 \%$ dos trabalhos encontrados, e $16 \%$ para os empírico-analíticos. Tais diferenças evidenciadas podem estar relacionadas aos critérios do estudo e às especificidades da temática escolar. Nesse sentido, os principais assuntos abordados nos estudos foram relacionados aos aspectos didáticos e pedagógicos da Educação Física, trabalhos que buscam oferecer diferentes formas de abordar o principal objeto da Educação Física na escola: o movimento humano que se manifesta de diversas maneiras no cotidiano escolar, por meio de jogos, brincadeiras e até mesmo em um simples caminhar.

\section{Artigos em periódicos nacionais e internacionais}

Foi encontrado um total de 242 trabalhos referentes ao tema Educação Física escolar (Tabela 5) em periódicos nacionais. O periódico que apresentou o maior número de artigos foi a Revista Brasileira de Atividade Física e Saúde (44), seguido dos periódicos Movimento (43) e Revista Brasileira de Cineantropometria e Desempenho Humano (43).

Tabela 5. Artigos em periódicos da Educação Física

\begin{tabular}{l|c}
\hline Revista & Artigos \\
\hline Revista Brasileira de Atividade Física e Saúde & 44 \\
\hline Movimento & 43 \\
\hline Revista Brasileira de Cineantropometria e Desempenho Humano & 43 \\
\hline Motriz: Revista de Educação Física & 41 \\
\hline Revista Brasileira de Ciências do Esporte & 34 \\
\hline Revista da Educação Física/UEM & 29 \\
\hline Revista Brasileira de Ciência e Movimento & 6 \\
\hline Revista Brasileira de Educação Física e Esporte & 2 \\
\hline TOTAL & $\mathbf{2 4 2}$ \\
\hline
\end{tabular}


Em relação à produção de artigos, nota-se um crescimento expressivo no número de publicações a partir do ano 2001 (Figura 2), acompanhando a expansão dos programas de pós-graduação stricto sensu e o aumento no número de dissertações e teses. Esses dados acompanham os resultados do estudo de Antunes et al. (2005), que analisou os resumos de artigos em periódicos nacionais e internacionais com a temática Educação Física escolar e constatou um aumento no número de artigos no período 1999-2003.

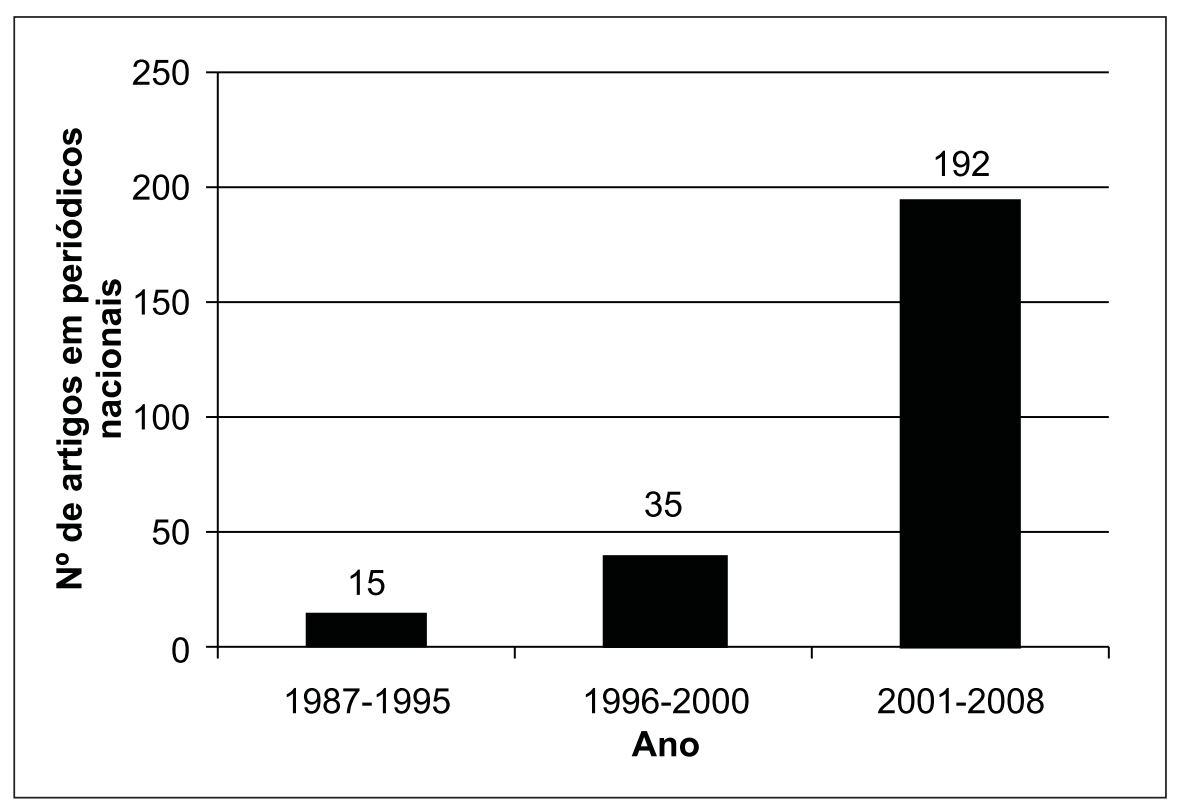

Figura 2. Artigos publicados (1987-2010)

Quanto aos temas dos trabalhos, constatou-se um predomínio de artigos relacionados à saúde, seguido de cultura corporal de movimento, prática pedagógica, esporte na escola, entre outros (Tabela 6). Os estudos sobre o assunto saúde, em sua maioria, por meio de diferentes técnicas de medidas e avaliação, buscaram avaliar índices de sobrepeso e obesidade, além de possiveis patologias associadas. Já a cultura corporal do movimento está fundamentada na teoria críticosuperadora, uma vez que essa proposta entende que o objeto da área de conhecimento Educação Física é a cultura corporal que se concretiza nos seus diferentes temas, quais sejam, o esporte, a ginástica, o jogo, as lutas, a dança e a mímica, sistematizando o conhecimento da disciplina em ciclos (1. organização da identidade dos dados da realidade; 2 . iniciação à sistematização do conhecimento; 3. ampliação da sistematização do 
conhecimento; e 4. aprofundamento da sistematização do conhecimento). Assim, essa proposta entende que o conteúdo da Educação Física deva ser tratado de forma historicizada, de maneira a ser apreendido em seus movimentos contraditórios (BRACHT, 1999).

Tabela 6. Assuntos abordados nas publicações

\begin{tabular}{l|r|r}
\hline Tema & Artigos & \multicolumn{1}{|c}{$\%$} \\
\hline Saúde & 87 & $36 \%$ \\
\hline Cultura corporal de movimento & 40 & $17 \%$ \\
\hline Prática pedagógica & 38 & $16 \%$ \\
\hline Esporte escolar & 18 & $7 \%$ \\
\hline Formação de professores & 17 & $7 \%$ \\
\hline Concepções sobre as aulas de Educação Física & 16 & $7 \%$ \\
\hline Desenvolvimento Humano & 14 & $6 \%$ \\
\hline Desempenho motor & 5 & $2 \%$ \\
\hline Gênero & 3 & $1 \%$ \\
\hline Interdisciplinaridade & 2 & $1 \%$ \\
\hline Memória Educação Física & 2 & $1 \%$ \\
\hline Total & $\mathbf{2 4 2}$ & $\mathbf{1 0 0 \%}$ \\
\hline
\end{tabular}

Além disso, ao classificar os conteúdos dos artigos, foi identificado que a maioria dos trabalhos utiliza métodos qualitativos (67\%) amparados nas Ciências Sociais e Humanas para analisar os dados. Aqueles que empregaram métodos quantitativos de análise vinculados às Ciências Naturais e Exatas somaram 33\%, acompanhando o que foi encontrado nas teses e dissertações.

Em nossa busca por artigos em periódicos internacionais, foram encontrados 227.418 relativos à Educação Física escolar, sendo 5,08\% referentes à Educação Física escolar no Brasil. Entretanto, sabemos que um mesmo artigo pode estar presente em mais de um banco de dados, podendo, assim, ocorrer sobreposição de dados (Tabela 7). A base de dados que apresentou o maior número de resultados foi a Scirus e depois a PubMed, a Scopuse a ISI Web of Knowledge. A base ScienceDirect não apresentou nenhum trabalho relacionado ao Brasil. 
Tabela 7. Artigos em bases de dados internacionais

\begin{tabular}{l|r|r}
\hline \multicolumn{1}{c|}{ Base de Dados } & Educação Física escolar & $\begin{array}{c}\text { Educação Física escolar } \\
\text { Brasil }\end{array}$ \\
\hline PubMed & 11.196 & 256 \\
\hline ScienceDirect & 576 & - \\
\hline Scirus & 204.401 & 11.221 \\
\hline ISI Web of Knowledge & 3.509 & 19 \\
\hline Scopus & 7.736 & 71 \\
\hline TOTAL & $\mathbf{2 2 7 . 4 1 8 ^ { * }}$ & $\mathbf{1 1 . 5 6 7 ^ { * }}$ \\
\hline
\end{tabular}

*Um mesmo artigo pode estar presente em mais de um banco de dados.

A pesquisa aqui realizada permitiu identificar a possibilidade da superação dos discursos dualistas que, de acordo com Betti (2005), dividem o campo teórico da Educação Física em dois grandes grupos: a matriz científica, que concebe a Educação Física como área de conhecimento científico, e a matriz pedagógica, que a concebe como prática pedagógica, como prática social de intervenção. Esse dualismo vem historicamente impondo alguns obstáculos para a evolução dos discursos e das práticas, concomitantemente, desvalorizando a concepção da Educação Física enquanto disciplina do currículo escolar.

A história da Educação Física tem acompanhado os momentos políticos, sociais e econômicos da sociedade, tendo suas raízes fortemente marcadas pelo caráter biologicista. Essa abordagem, de acordo com um coletivo de autores (1992), objetiva desenvolver e fortalecer física e moralmente os indivíduos, por meio de métodos rígidos compostos por séries de exercícios elaboradas a partir das ciências biológicas, o que lhe conferia um caráter científico. Conforme Bracht (1999), o nascimento da Educação Física se deu, por um lado, para cumprir a função de colaborar na construção de corpos saudáveis e dóceis, ou melhor, com uma educação estética que permitisse uma adequada adaptação ao processo produtivo ou a uma perspectiva política nacionalista, e, por outro, foi também legitimado pelo conhecimento médico-científico do corpo, que referendava as possibilidades, a necessidade e as vantagens de tal intervenção sobre o corpo; essas marcas persistem até hoje. Além disso, os dados do presente estudo demonstram que a Educação 
Física enquanto área de conhecimento é muito abrangente, pois estuda o fenômeno do movimento humano, desde o seu nivel de análise bioquímico até o sociocultural (ANTUNES et al., 2005).

Acerca das diferenças entre as linhas de pesquisa da Educação Física, Betti et al. (2004) argumentam que, algumas vezes, a pesquisa pedagógica e sociocultural tende a produzir trabalhos de natureza local e, portanto, de pouco interesse para publicações internacionais. Já as áreas Exatas e Biológicas têm maior facilidade para a inserção internacional, por tratarem de fenômenos do mundo físico/biológico e buscarem leis/ explicações universais - isso justifica em parte o maior número de linhas de estudo voltadas para a temática atividade física e saúde.

Assim, devido à amplitude de atuação do professor de Educação Física, Lüdorf (2002) aponta que a Educação Física, ao buscar as bases científicas para fundamentar sua prática, pode selecionar tanto estudos oriundos das Ciências Naturais Exatas, voltados ao ensino e aprimoramento de técnicas específicas, ao treinamento e à preparação física de modalidades esportivas, quanto das Ciências Humanas e Sociais, que se relacionam com as questões pedagógicas, culturais, antropológicas, filosóficas e sociais do movimento humano.

\section{Considerações finais}

A partir dos resultados desta pesquisa, pode-se destacar o aumento das bases de produção científica da Educação Física nos quatro aspectos avaliados: grupos de pesquisa, programas de pós-graduação stricto sensu, teses e dissertações e artigos científicos sobre a temática educação em todas as regiões do Brasil, destacando as regiões Sul e Sudeste como os principais centros de produção de conhecimento. Foram identificadas também as instituições públicas de ensino superior como os principais centros de pesquisa da Educação Física nos aspectos avaliados pelo estudo.

Essa ampliação da base de sustentação da pesquisa em Educação Física é acompanhada por um grande contingente de pesquisadores envolvidos. Sobre os grupos de pesquisa, identificamos 
um amplo rol de objetos, por vezes bastante distintos, que variam entre estudos relacionados à atividade física e à saúde, a aspectos pedagógicos e didáticos da Educação Física escolar, ao esporte e ao alto rendimento, entre outros. Acreditamos que um dos principais motivos para esses resultados é o vasto campo de possibilidades de atuação do professor de Educação Física.

Com relação aos programas de pós-graduação e à produção de teses e dissertações, podemos verificar quanto às bases teóricas e metodológicas que os estudos sobre a Educação Física escolar se diferenciam quando comparados aos do campo da Educação Física de modo geral, pois essa linha de pesquisa apresenta um acréscimo no número de investigações de cunho qualitativo, correspondendo a mais de $80 \%$ dos estudos encontrados. 0 mesmo também foi identificado nos artigos em periódicos nacionais indexados. Porém o tema mais recorrente, nesse item, foi saúde, sendo tratado principalmente sob a ótica diagnóstica, ou seja, o levantamento de dados e as possíveis associações com diferentes patologias. Desse modo, constatou-se que, mesmo havendo o predomínio das linhas de pesquisa ligadas à atividade física e à saúde nos programas de pós-graduação, as pesquisas atreladas ao cotidiano escolar vêm conquistando um espaço cada vez maior, ampliando os horizontes metodológicos e epistemológicos da Educação Física, abrindo espaço para a superação dos discursos dualistas que dificultam o avanço conjunto de teoria e prática.

Contudo, após este trabalho, encontramos pontos que devem ser melhorados, pois o número de pesquisas vem aumentando significativamente e muitos dos programas de pós-graduação não mantêm seus domínios eletrônicos atualizados, o que dificulta o acesso à produção acadêmica para o público em geral. Da mesma forma, há a necessidade de uma maior aproximação entre os centros de produção do conhecimento (universidades) e os locais de aplicação desses conhecimentos (escolas), por meio de parcerias que propiciem o desenvolvimento de atividades de ensino, pesquisa e extensão. Acreditamos que tal relacionamento colaborativo pode beneficiar tanto as ações da universidade (qualificando e adequando as pesquisas à realidade escolar) quanto favorecer uma melhoria da qualidade do ensino nas escolas por meio da capacitação dos educadores. 
Sobre o impacto das pesquisas sobre o contexto escolar nos programas de pós-graduação da Educação Física, pode-se afirmar que esses trabalhos possuem um efeito renovador sobre a produção científica da área, possibilitando a atualização e reciclagem de conteúdos; com isso, é reforçado o papel da Educação Física como área de produção de conhecimento. Além do mais, os resultados desses trabalhos podem fornecer subsídios para o desenvolvimento de estratégias tanto no ensino superior quanto para a educação básica, estreitando os vínculos entre escola e universidade.

Portanto, espera-se que este estudo possa servir para orientar decisões estratégicas para o desenvolvimento do sistema nacional de pós-graduação como um todo (KOKUBUN, 2003), devido ao reconhecimento da necessidade, por parte dos governos e da comunidade científica nacional, da importância de pesquisas que apontem indicadores da produtividade científica e que sirvam para definição de diretrizes, alocação de investimentos e recursos, formulação de programas e avaliação de atividades relacionadas ao desenvolvimento científico e tecnológico no País (MUGNAINI; JANNUZZI; QUONIAM, 2004). Ademais, com um maior número de estudiosos envolvidos com a temática educacional, aumenta a possibilidade de que sejam superados os desencontros existentes entre a teoria e a prática, rompendo com a barreira divisória entre o mundo dos pesquisadores acadêmicos e dos professores nas escolas. Assim, esperamos que o presente estudo seja mais uma ferramenta para a reflexão, contribuindo para a melhoria da produção acadêmica da Educação Física voltada ao contexto escolar.

Recebido em 9/10/2010

Aprovado em 24/8/2011

\section{Agradecimentos}

Capes, CNPq, Fapergs e Finep. 


\section{Referências bibliográficas}

AMADIO, A. C. Trajetória da pós-graduação stricto sensu na escola de Educação Física e esporte da universidade de São Paulo após 25 anos de produção acadêmica. Revista Brasileira de Ciências do Esporte, Campinas, v. 24, n. 2, p. 27-47, jan. 2003.

ANTUNES, F. H. C. et al. Um retrato da pesquisa brasileira em Educação Física escolar: 1999 - 2003. Motriz, Rio Claro, v. 11, n. 3, p. 179-184, set./ dez. 2005.

BAPTISTA, M. T. et al. Influência da Escola de Educação Física do Exército na origem do currículo de Educação Física no Brasil. Revista de Educação Física, n. 126, p. 10-14, 2002.

BELTRAMI, D. M. Dos fins da Educação Física escolar. Revista da Educação Física/UEM, Maringá, v. 12, n. 2, p. 27-33, 2001.

BETTI, M. Educação Física como prática científica e prática pedagógica: reflexões à luz da filosofia da ciência. Revista Brasileira de Educação Física e Esporte, São Paulo, v. 19, n. 3, p. 183-97, jul./set. 2005.

BETTI, M. et al. A avaliação da Educação Física em debate: implicações para a subárea pedagógica e sociocultural. Revista Brasileira de Pósgraduação, v. 1, n. 2, p. 183-194, nov. 2004.

BRACHT, V. A constituição das teorias pedagógicas da Educação Física. Cadernos Cedes, n. 48, ano XIX, ago. 1999.

CAPARROZ, F. E.; BRACHT, V. O tempo e o lugar de uma didática da Educação Física. Revista Brasileira de Ciências do Esporte, Campinas, v. 28, n. 2, p. 21-37, jan. 2007.

CAPES. Relatório de Avaliação 2007-2009. Disponivel em: < http:/ / www. capes.gov.br/>. Acesso em: 27 set. 2010.

CARVALHO, J. M. Os espaços/tempos da pesquisa sobre o professor. Educação e Pesquisa, São Paulo, v. 28, n. 2, p. 69-86, jul./dez. 2002. 
CARVALHO, L. M.; TOMAZELLO, M. G. C.; OLIVEIRA, H. T. Pesquisa em educação ambiental: panorama da produção brasileira e alguns de seus dilemas. Cadernos CEDES (on-line), v. 29, n. 77, p. 13-27, 2009.

COLETIVO DE AUTORES. Metodologia do Ensino de Educação Física. 2. ed. São Paulo: Cortez, 1992.

DAOLIO, J. O Ser e o Tempo da pesquisa sociocultural em Educação Física. Revista Brasileira de Ciências do Esporte, Campinas, v. 29, n. 1, p. 49-60, set. 2007.

DE MEIS, L.; ARRUDA, A. P.; GUIMARÃES, J. The Impact of Science in Brazil. IUBM Life, v. 59, n. 4, p. $227 \quad$ 234, 2007.

FALCÃO, J. L. C. A produção do conhecimento na Educação Física brasileira e a necessidade de diálogos com os movimentos da cultura popular. Revista Brasileira de Ciências do Esporte, Campinas, v. 29, n. 1, p. 143-161, set. 2007.

FERON, A. V.; MORAES E SILVA, M. A igreja do "diabo" e a produção de conhecimento em Educação Física. Revista Brasileira de Ciências do Esporte, Campinas, v. 29, n. 1, p. 107-122, set. 2007.

GAMBOA, S. S.; CHAVES, M.; TAFFAREL, C. A pesquisa em Educação Física no nordeste brasileiro (Alagoas, Bahia, Pernambuco e Sergipe), 19822004. Revista Brasileira Ciências do Esporte, Campinas, set. 2007.

GOMES, U. Avaliação da produção científica do departamento de bioquímica da Universidade Federal do Rio Grande do Sul. 2007. Dissertação (Mestrado em Educação em Ciências) - Programa de Pós-Graduação em Educação em Ciências: Química da Vida e Saúde, Universidade Federal do Rio Grande do Sul, Porto Alegre.

KOKUBUN, E. Pós-graduação em educação física no Brasil: indicadores objetivos dos desafios e das perspectivas. Revista Brasileira de Ciências do Esporte, v. 24, n. 2, p. 9-26, 2003. 
LETA, J. As mulheres na ciência brasileira: crescimento, contrastes e um perfil de sucesso. Estudos Avançados (on-line), v. 17, n. 49, p. 271-284, 2003.

LÜDORF, S. M. A. Panorama da pesquisa em Educação Física da década de 90: Análise dos resumos de dissertações e teses. Revista da Educa Física/UEM, Maringá, v. 13, n. 2, p. 19-25, 2002.

MARTINS, N. R.; SILVA, R. V. S. Pesquisas brasileiras em Educação Física e Esportes: tendências das teses e dissertações. Disponivel em: <http:/ / www.nuteses.ufu.br/trabalho_2.pdf>. Acesso em: 20 jan. 2009.

MARZARI, J.; ACOSTA, M. A. F. Diagnóstico da produção científica na temática terceira idade no período 2001-2006. Revista Brasileira de Ciências do Esporte, Campinas, v. 29, n. 1, p. 123-141, set. 2007.

MELO, V. A. Reflexão sobre a História da Educação Física no Brasil: Uma abordagem historiográfica. Movimento, n. 4, ano III, 1996.

MOUTINHO, K.; CUNHA FILHO, P. Líber: Alternativa para publicação eletrônica. Ciência da Informação, Brasília, v. 31, n. 2, p. 80-85, maio/ ago. 2002.

MUGNAINI, R.; JANNUZZI, P. M.; QUONIAM, L. Indicadores bibliométricos da produção científica brasileira: uma análise a partir da base Pascal. Ciência e Informação, v. 33, n. 2, p. 123-131, 2004.

PRADO, S. D.; AMORIM, A. E.; ABREU, C. Centro de Referência e Documentação sobre Envelhecimento UnATI-UERJ: identificação, sistematização e disseminação de informações sobre envelhecimento humano no Brasil. Textos sobre Envelhecimento (Uerj), Rio de Janeiro, v. 6, n. 1, p. 61-79, 2003

QUINTÃO F. A.; VAZ, A. F. Do giro lingüístico ao giro ontológico na atividade epistemológica em Educação Física. Movimento, v. 16, n. 3, p. 11-29, jul./set. 2010. 
SMITH, A. apud VANTI, N. A. P. Da bibliometria à webometria: uma exploração conceitual dos mecanismos utilizados para medir o registro da informação e a difusão do conhecimento. Ciência da Informação, Brasília, v. 31, n. 2, p. 1-15, maio/ago. 2002.

SOBRAL, F. A. F. Qualidade acadêmica e relevância social e econômica da educação superior. Cadernos Cedes (on-line), v. 29, n. 78, p. 227-241, 2009.

SOUSA, J. V. Qualidade na educação superior: lugar e sentido na relação público-privado. Cadernos Cedes (on-line), v. 29, n. 78, p. 242-256, 2009.

SOUZA, A. P. T.; ISAYAMA, H. F. Lazer e Educação Física: análise dos grupos de pesquisa em lazer cadastrados na plataforma LATTES do CNPQ. EFdeportes Revista Digital, Buenos Aires, n. 99, ano 11, ago. 2006.

SOUZAE SILVA, R. V. Mestrados em educação física no Brasil: pesquisando suas pesquisas. 1990. Dissertação (Mestrado em Ciência do Movimento Humano) - Programa de Pós-Graduação em Ciência do Movimento Humano, Universidade Federal de Santa Maria, Santa Maria.

TANI, G. Os desafios da pós-graduação em Educação Física. Revista Brasileira de Ciências do Esporte, v. 22, n. 1, p. 79-90, set. 2000.

VAGO, T. M. Início e fim do século XX: maneiras de fazer Educação Física na escola. Cadernos Cedes (on-line), v. 19, n. 48, p. 30-51, 1999. 
RBPG 
Área de radiofarmácia do Instituto de Pesquisas Energéticas e Nucleares (Ipen),

em 1973, onde atualmente são produzidos elementos radioativos utilizados para diagnósticos e terapias em medicina nuclear. Foto do Arquivo do Ipen.

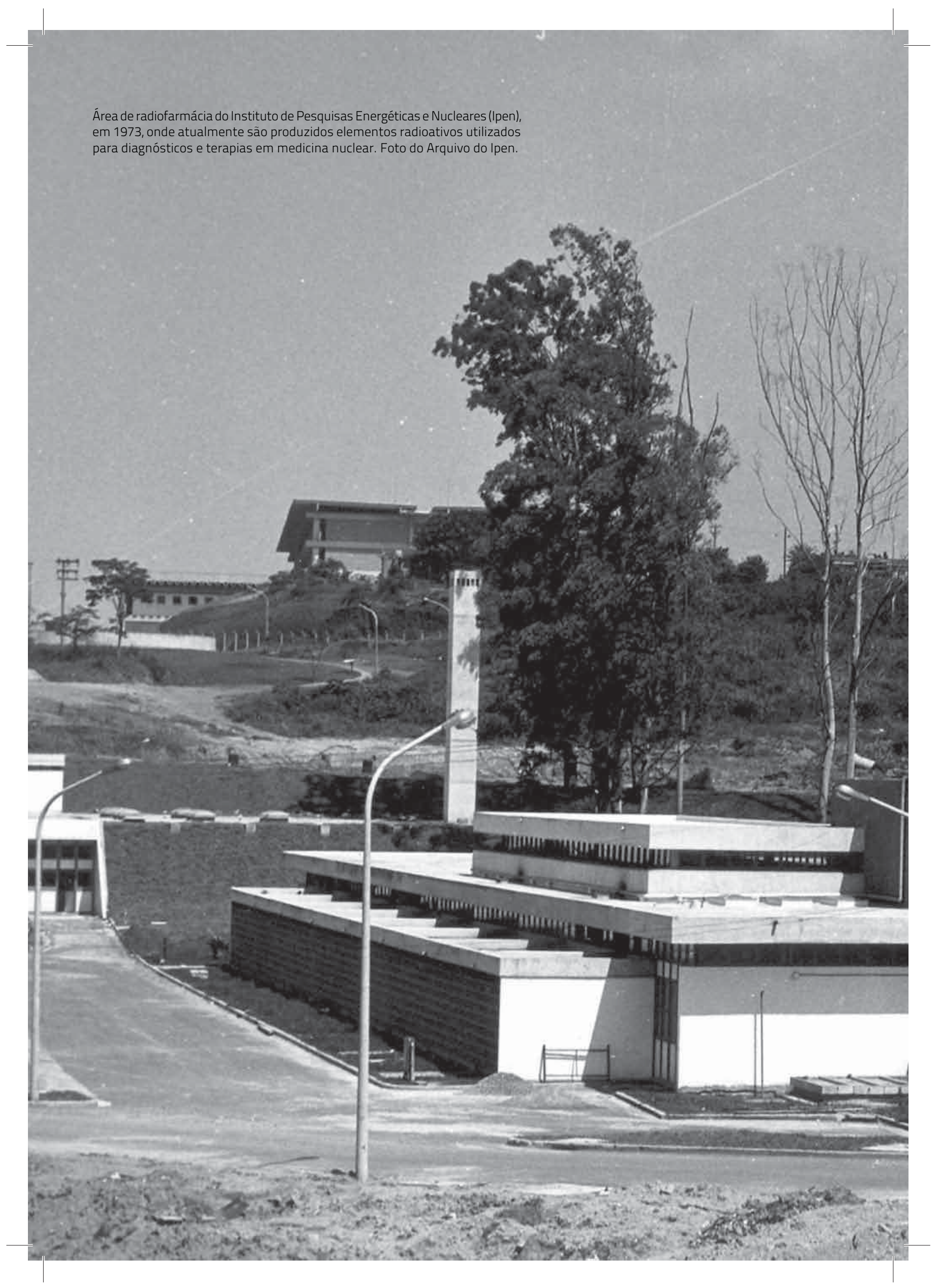




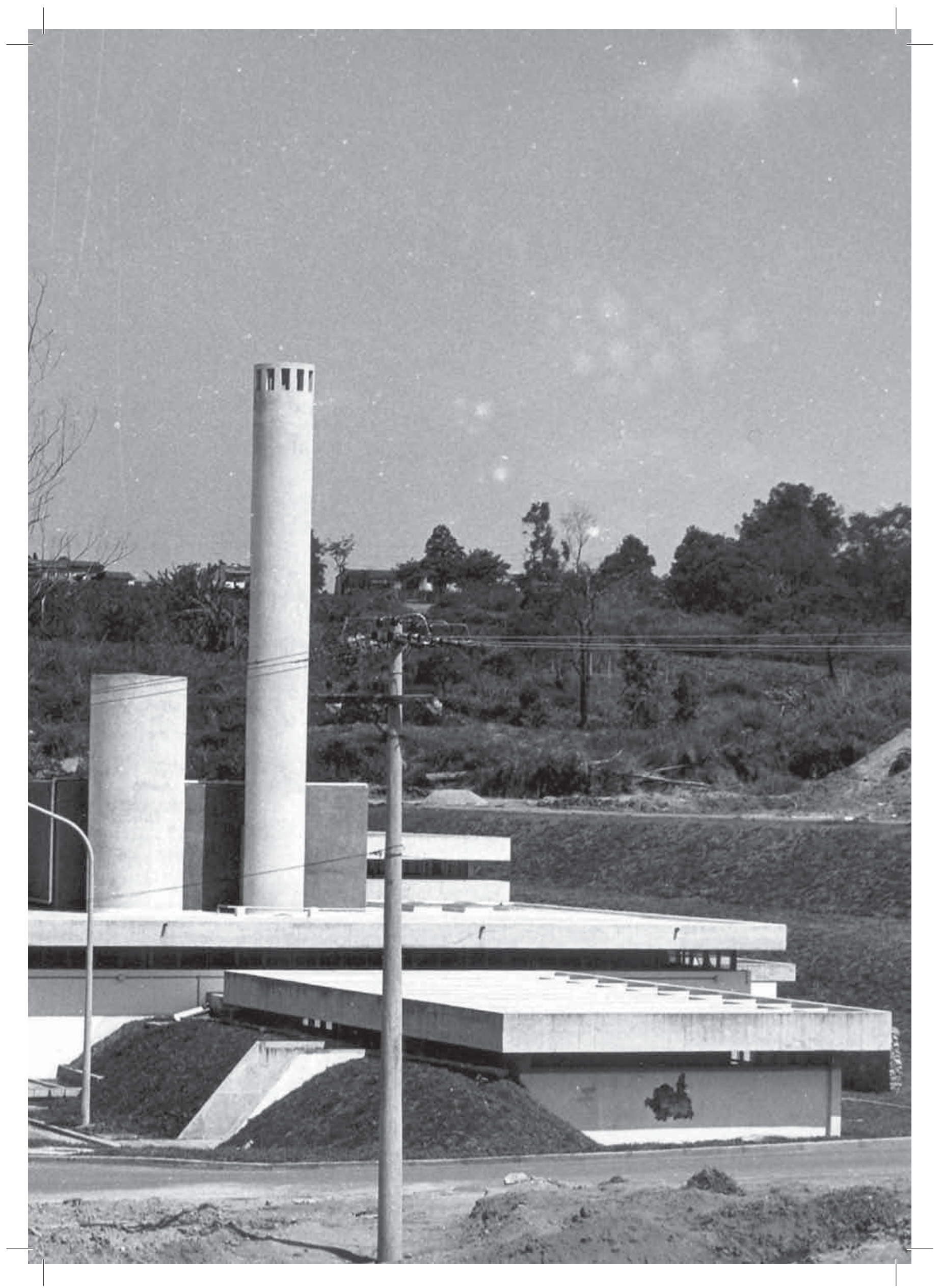


